\title{
Comparison study of some finite volume and finite element methods for the shallow water equations with bottom topography and friction terms
}

\author{
M. Lukáčová - Medvidová ${ }^{1}$ and U. Teschke ${ }^{2}$
}

\begin{abstract}
We present a comparison of two discretization methods for the shallow water equations, namely the finite volume method and the finite element scheme. A reliable model for practical interests includes terms modelling the bottom topography as well as the friction effects. The resulting equations belong to the class of systems of hyperbolic partial differential equations of first order with zero order source terms, the so-called balance laws. In order to approximate correctly steady equilibrium states we need to derive a well-balanced approximation of the source term in the finite volume framework. As a result our finite volume method, a genuinely multidimensional finite volume evolution Galerkin (FVEG) scheme, approximates correctly steady states as well as their small perturbations (quasi-steady states). The second discretization scheme, which has been used for practical river flow simulations, is the finite element method (FEM). In contrary to the FVEG scheme, which is a time explicit scheme, the FEM uses an implicite time discretization and the NewtonRaphson iterative scheme for inner iterations. We compare the accuracy and performance of both scheme through several numerical experiments, which demonstrate the reliability of both discretization techniques and correct approximation of quasisteady states with bottom topography and friction.
\end{abstract}

Key words: well-balanced schemes, steady states, systems of hyperbolic balance laws, shallow water equations, evolution Galerkin schemes, finite element schemes, Darcy-Weisbach friction law, Newton-Raphson method

AMS Subject Classification: 65L05, 65M06, 35L45, 35L65, 65M25, 65M15

\section{Introduction}

Description of natural river processes is very complex. The main aim is to determine the water level at a specific place and time. Reliable mathematical models as well as robust, fast and accurate numerical simulations are very important for predictions of floods and have large economical impact. One of the main difficulty of the reliable calculation is the determination of the friction which counteracts the river flows. Numerical simulation of

\footnotetext{
${ }^{1}$ Department of Mathematics, Hamburg University of Technology, Schwarzenbergstraße 95, 21079 Hamburg, Germany, email: lukacova@tu-harburg.de

${ }^{2}$ Institut for River and Coastal Engineering, Hamburg University of Technology, Denickestraße 22, 21073 Hamburg, Germany, email: teschke@tu-harburg.de
} 
natural river flows is based on the two-dimensional shallow water equations. The shallow water system consists of the continuity equation and the momentum equations

$$
\frac{\partial \boldsymbol{u}}{\partial t}+\frac{\partial \boldsymbol{f}_{1}(\boldsymbol{u})}{\partial x}+\frac{\partial \boldsymbol{f}_{2}(\boldsymbol{u})}{\partial y}=\boldsymbol{b}(\boldsymbol{u})
$$

where

$$
\begin{aligned}
\boldsymbol{u} & =\left(\begin{array}{c}
h \\
h u \\
h v
\end{array}\right), \boldsymbol{f}_{1}(\boldsymbol{u})=\left(\begin{array}{c}
h u \\
h u^{2}+\frac{1}{2} g h^{2} \\
h u v
\end{array}\right) \\
\boldsymbol{f}_{2}(\boldsymbol{u}) & =\left(\begin{array}{c}
h v \\
h u v \\
h v^{2}+\frac{1}{2} g h^{2}
\end{array}\right), \boldsymbol{b}(\boldsymbol{u})=\left(\begin{array}{c}
0 \\
-g h\left(\frac{\partial b}{\partial x}+S_{f x}\right) \\
-g h\left(\frac{\partial b}{\partial y}+S_{f y}\right)
\end{array}\right) .
\end{aligned}
$$

Here $h=h(x, y)$ denotes the water depth, $u=u(x, y, t), v=(x, y, t)$ are vertically averaged velocity components in $x$ - and $y$-direction, $g$ stands for the gravitational constant, $b=b(x, y)$ denotes the bottom topography and $S_{f x}, S_{f y}$ are the friction terms in $x-$ and $y-$ directions.

In practice even the one dimensional analogy of (1.1), the so-called Saint-Venant equations, are often used

$$
\frac{\partial \boldsymbol{w}}{\partial t}+\frac{\partial \boldsymbol{f}_{1}(\boldsymbol{w})}{\partial x}=\boldsymbol{b}(\boldsymbol{w})
$$

where

$$
\boldsymbol{w}=\left(\begin{array}{c}
A \\
Q
\end{array}\right), \boldsymbol{f}_{1}(\boldsymbol{u})=\left(\begin{array}{c}
Q \\
Q^{2} / A
\end{array}\right), \boldsymbol{b}(\boldsymbol{w})=\left(\begin{array}{c}
0 \\
-g A\left(\frac{\partial z}{\partial x}+S_{f x}\right)
\end{array}\right) .
$$

Here $A=A(h(x, t), x)$ denotes the cross section area, $Q=Q(x, t)=A u$ is the discharge and $z=h+b$ stands for the water surface.

The determination of the friction slopes $S_{f x}, S_{f y}$ is a very complex problem. The friction law for the river flow is often approximately modelled by the friction law of pipe flow, but the pipe flow is much simpler than natural river flow. In fact, the main difficulty of the evaluation of friction slope is the reflection of various characteristics of natural river flow into one parameter. Typical characteristics of natural rivers are:

- structured cross sectional area with mass and momentum exchange at the boundaries

- complex cross sectional area as a function of depth of water

- vegetation of different kind

- different roughness at the same cross sectional area

- meandering

- retention effects.

A good overview of the theory of friction slope in natural river flow can be found in [17] and the references therein. For one dimensional steady flow situation $S_{f x}$ can be expressed by an integral relation

$$
\rho g \int_{x_{1}}^{x_{2}} S_{f x} A(h(x), x) \mathrm{d} x=\int_{A_{b o t}} \tau_{b o t}(x) \mathrm{d} x \mathrm{~d} y .
$$


The right hand side term describes a part of the weight of the fluid element with the cross-section $A(x)$. Here $\tau_{b o t}$ is the shear stress at the river bottom at the bottom area $A_{b o t}$ and $x_{1}$ and $x_{2}$ are the boundaries of the fluid element in the $x$-direction. The bottom composition of a river can vary rapidly, especially when vegetation is taken into account. In the literature several methods in order to determine the friction slope can be found, cf., e.g. [16] and [19]. Basis for our calculation is the friction law of Darcy-Weisbach. Thus, the friction slopes are calculated by, see, e.g. [17],

$$
S_{f x}(h, u, v, x, y, t)=\frac{\lambda u \sqrt{u^{2}+v^{2}}}{8 g h}, \quad S_{f y}(h, u, v, x, y, t)=\frac{\lambda v \sqrt{u^{2}+v^{2}}}{8 g h},
$$

where $\lambda$ stays for the so-called resistance value. This is determined according to the simplified form of the Colebrook-White relation

$$
\frac{1}{\sqrt{\lambda}}=-2.03 \log \left(\frac{k_{s} / h}{14.84}\right)
$$

which was originally found for pipe flow. In the case of one-dimensional flow the friction slope $S_{f}$ is given in the analogy to (1.3) as

$$
S_{f}(A, Q, x, t)=\frac{\lambda}{8 g r_{h y}} \frac{|Q| Q}{A}, \quad \frac{1}{\sqrt{\lambda}}=-2.03 \log \left(\frac{k_{s} / r_{h y}}{14.84}\right),
$$

where $r_{h y}$ stays for the hydraulic radius. When the above listed characteristics of natural rivers have to be reflected more complex models for the resistance value $\lambda$ are necessary. Here $k_{s}$ denotes the Nikuradse grain roughness size, which depends on the composition of the river bottom, especially of the sediment size. Typically, $k_{s}$ can vary from $1 \mathrm{~mm}$ for beton until $300 \mathrm{~mm}$ for bottom with dense vegetation, or sometimes in an even wider range.

One possible and simple way to solve a system of balance laws (1.1) or (1.2) is to apply the operator splitting approach and solve separately the resulting homogenous system of hyperbolic conservation laws, e.g. by using the finite volume or finite element method, and the system of ordinary differential equations, which includes the right-hand-side source terms. However, this can lead to the structural deficiencies and strong oscillations in the solutions, especially when steady solutions or their small perturbations are to be computed numerically. In fact, most of the geophysical flows, including river flows, are nearly steady flows, that are closed to the equilibrium states of the dynamical system (1.1). In this case the gradient of fluxes is balanced with the right-hand side source term, i.e. we have the following balance condition in the $x$-direction $\partial_{x}\left(g h^{2} / 2\right)=-g h\left(\partial_{x} b+S_{f x}\right)$. Assume that $R$ is a primitive to $S_{f x}$. Then the balance condition can be rewritten as $g h \partial_{x}(h+b+R)=0$. An analogous condition holds in the $y$ - direction. These equilibrium conditions yield the well-balanced approximation of the source term. The resulting schemes are called the well-balanced schemes, cf., e.g. [1], [4], [5], [7] and the references therein for other well-balanced schemes in literature.

Our aim is to study the approximation of steady equilibrium states for balance law (1.1), or (1.2), in the framework of the finite element as well as finite volume methods. In the case of the finite volume method we use a genuinely multidimensional finite volume evolution Galerkin scheme, which has been shown to perform very accurate in comparison to classical finite volume methods, e.g. dimensional splitting schemes, cf. [9], [10]. 


\section{Finite element method}

The finite element method is a well known method for solving differential equations. Numerous research and applications have shown good results in the area of structural as well as fluid mechanics. Our approach uses a formulation based on the method of weighted residuals to develop the discrete equations.

The method presented here has been used for practical applications in hydrology. Let $q_{e}$ be the lateral inflow per unit length and $\beta$ denote the momentum coefficient for flows with the velocity, which is not uniform, i.e.

$$
\beta=\frac{A}{Q^{2}} \int_{A} u^{2}(y, z) \mathrm{d} x \mathrm{~d} y .
$$

Then the continuity equation $(1.2)_{1}$ is equivalent to

$$
\frac{\partial A}{\partial t}+\frac{\partial Q}{\partial x}-q_{e}=0
$$

Applying the rule for derivation of fraction $Q^{2} / A$ in $(1.2)_{2}$ we obtain the following formulation of the momentum equation, which is equivalent to $(1.2)_{2}$ for smooth solutions

$$
\frac{\partial Q}{\partial t}+\frac{Q^{2}}{A} \frac{\partial \beta}{\partial x}+2 \beta \frac{Q}{A} \frac{\partial Q}{\partial x}-\beta \frac{Q^{2}}{A^{2}} \frac{\partial A}{\partial x}+g A \frac{\partial z}{\partial x}+g A S_{f}-q_{e} v_{e x}=0
$$

Here $v_{e x}$ is the velocity component of the inflow in the $x$-direction. For the detailed derivation of (2.2) and (2.3) see [17].

The finite element approximation with the basis functions $N_{i}(x)$ for the independent variables $h$ and $Q$ gives

$$
Q(x)=\sum_{i=1}^{n} Q_{i} N_{i}(x), \quad h(x)=\sum_{i=1}^{n} h_{i} N_{i}(x),
$$

where $i$ is the index of a node, $n$ is the total number of nodes, $h_{i}$ approximates the water depth and $Q_{i}$ the discharge at the node $i$. The cross-section $A(h, x)$ is a given function depending on $h$ and $x$. The differential equations (2.2) and (2.3) are weighted with weighted functions (i.e. test functions) over the whole domain $\Omega$ yielding the following equations

$$
\begin{gathered}
G \equiv \int_{\Omega} W_{i}\left(\frac{\partial A}{\partial t}+\frac{\partial Q}{\partial x}-q_{e}\right) \mathrm{d} x=0, \quad i=1,2, \ldots, n \\
F \equiv \int_{\Omega} W_{i}\left(\frac{\partial Q}{\partial t}+\frac{Q^{2}}{A} \frac{\partial \beta}{\partial x}+2 \beta \frac{Q}{A} \frac{\partial Q}{\partial x}-\beta \frac{Q^{2}}{A^{2}} \frac{\partial A}{\partial x}+g A \frac{\partial z}{\partial x}+g A S_{f}-q_{e} v_{e x}\right) \mathrm{d} x=0 \\
i=1,2, \ldots, n .
\end{gathered}
$$

We have chosen the weighted functions $W_{i}(x)$ to be the same functions as the basis function $N_{i}(x)$. Equations (2.5) and (2.6) represent the classical Galerkin Method. 


\subsection{Time integration scheme}

In the time integration scheme we follow an approach of King [11]. The variation with time will be described by the following function

$$
y(t)=a+b t+c t^{\gamma}
$$

with a constant coefficient $\gamma$. It can be shown that the following equation

$$
\frac{\mathrm{d} y(t+\Delta t)}{\mathrm{d} t}=\gamma \frac{y(t+\Delta t)-y(t)}{\Delta t}+(1-\gamma) \frac{\mathrm{d} y(t)}{\mathrm{d} t} .
$$

holds [17]. In our numerical experiments for steady or quasi-steady, i.e. perturbed steady flows, we have tested several values of $\gamma, \gamma \in[1,2]$, and found only marginal differences. Therefore we set $\gamma=1$. In this case the scheme reduces to the conventional linear integration scheme, i.e. the implicit Euler method.

\subsection{Newton Raphson Procedure}

Since the equations (2.5) and (2.6) are nonlinear we have used the Newton-Raphson procedure in order to solve them iteratively

$$
\left[\begin{array}{cccccc}
\frac{\partial F_{1}}{\partial h_{1}} & \cdots & \frac{\partial F_{1}}{\partial h_{n}} & \frac{\partial F_{1}}{\partial Q_{1}} & \cdots & \frac{\partial F_{1}}{\partial Q_{n}} \\
\frac{\partial G_{1}}{\partial h_{1}} & \cdots & \frac{\partial G_{1}}{\partial h_{n}} & \frac{\partial G_{1}}{\partial Q_{1}} & \cdots & \frac{\partial G_{1}}{\partial Q_{1}} \\
\vdots & \ddots & \vdots & \vdots & \ddots & \vdots \\
\frac{\partial F_{n}}{\partial h_{1}} & \cdots & \frac{\partial F_{n}}{\partial h_{n}} & \frac{\partial F_{n}}{\partial Q_{1}} & \cdots & \frac{\partial F_{n}}{\partial Q_{n}} \\
\frac{\partial G_{n}}{\partial h_{1}} & \cdots & \frac{\partial G_{n}}{\partial h_{n}} & \frac{\partial G_{n}}{\partial Q_{1}} & \cdots & \frac{\partial G_{n}}{\partial Q_{n}}
\end{array}\right] \cdot\left[\begin{array}{c}
\left(h_{1}^{\text {new }}-h_{1}^{\text {old }}\right) \\
\vdots \\
\left(h_{n}^{\text {new }}-h_{n}^{\text {old }}\right) \\
\left(Q_{1}^{\text {new }}-Q_{1}^{\text {old }}\right) \\
\vdots \\
\left(Q_{n}^{\text {new }}-Q_{n}^{\text {old }}\right)
\end{array}\right]+\left[\begin{array}{c}
F_{1} \\
G_{1} \\
\vdots \\
\vdots \\
F_{n} \\
G_{n}
\end{array}\right]=\left[\begin{array}{c}
0 \\
0 \\
\vdots \\
\vdots \\
0 \\
0
\end{array}\right]
$$

Most of the derivatives in the Jacobian matrix are zeros, which is a consequence of the used basis functions, i.e. the Jacobian is a sparse matrix. Equations (2.9) have to be simplified further. The integrals $F_{1}, \ldots, F_{n}$ and $G_{1}, \ldots, G_{n}$ as well as their derivatives, cf. (6.2) - (6.6), have to be approximated by a suitable numerical integration. In our method we have used the Gauss quadrature rule with four points [11].

\section{Finite volume evolution Galerkin method}

In our recent works [8], [9], [10] we have proposed a new genuinely multidimensional finite volume evolution Galerkin method (FVEG), which is used to solve numerically nonlinear hyperbolic conservation laws. The method is based on the theory of bicharacteristics, which is combined with the finite volume framework. It can be also viewed as a predictorcorrector scheme; in the predictor step data are evolved along the bichracteristics, or along the bicharacteristic cone, in order to determine approximate solution on cell interfaces. In the corrector step the finite volume update is done. Thus, in our finite volume method we do not use any one-dimensional approximate Riemann solver, instead the intermediate solution on cell-interfaces is computed by means of an approximate evolution operator. The reader is referred to [3], [6], [14] and the references therein for other recent genuinely multidimensional methods.

To point out multidimensional features of the FVEG scheme we will give the description of the method for two-dimensional situations. Our computational domain $\Omega$ wil be divided 
into a finite number of regular finite volumes $\Omega_{i j}=\left[x_{i-\frac{1}{2}}, x_{i+\frac{1}{2}}\right] \times\left[y_{j-\frac{1}{2}}, y_{j+\frac{1}{2}}\right]=\left[x_{i}-\right.$ $\left.\hbar / 2, x_{i}+\hbar / 2\right] \times\left[y_{j}-\hbar / 2, y_{j}+\hbar / 2\right], i, j \in \mathbb{Z}, \hbar$ is a mesh step. Further, we denote by $\boldsymbol{U}_{i j}^{n}$ the piecewise constant approximate solution on a mesh cell $\Omega_{i j}$ at time $t_{n}$ and start with initial approximations obtained by the integral averages $\boldsymbol{U}_{i j}^{0}=\int_{\Omega_{i j}} \boldsymbol{U}(\cdot, 0)$. The finite volume evolution Galerkin scheme can be formulated as follows

$$
\boldsymbol{U}^{n+1}=\boldsymbol{U}^{n}-\frac{\Delta t}{\hbar} \sum_{k=1}^{2} \delta_{x_{k}} \overline{\boldsymbol{f}}_{k}^{n+1 / 2}+\boldsymbol{B}^{n+1 / 2}
$$

where $\Delta t$ is a time step, $\delta_{x_{k}}$ stays for the central difference operator in the $x_{k}$-direction, $k=1,2$, and $\overline{\boldsymbol{f}}_{k}^{n+1 / 2}$ represents an approximation to the edge flux at the intermediate time level $t_{n}+\Delta t / 2$. Further $\boldsymbol{B}^{n+1 / 2}$ stands for the approximation of the source term $\boldsymbol{b}$. The cell interface fluxes $\overline{\boldsymbol{f}}_{k}^{n+1 / 2}$ are evolved using an approximate evolution operator denoted by $E_{\Delta t / 2}$ to $t_{n}+\Delta t / 2$ and averaged along the cell interface edge denoted by $\mathcal{E}$, i.e.

$$
\overline{\boldsymbol{f}}_{k}^{n+1 / 2}:=\frac{1}{\hbar} \int_{\mathcal{E}} \boldsymbol{f}_{k}\left(E_{\Delta t / 2} \boldsymbol{U}^{n}\right) \mathrm{d} S
$$

The well-balanced approximate evolution operator $E_{\Delta t / 2}$ for system (1.1) will be given in the Section 3.2.

\subsection{A well-balanced approximation of the source terms in the finite volume update}

As already mentioned above we want to approximate source terms in the finite volume update in such a way that the balance between the source terms and the gradient of fluxes will be exactly preserved. This can be done by approximating the source term by using its values on interfaces, cf. [15].

Let us consider a steady flow,

$$
\begin{aligned}
& \frac{\mathrm{d} u}{\mathrm{~d} t} \equiv \frac{\partial u}{\partial t}+u \frac{\partial u}{\partial x}+v \frac{\partial u}{\partial y}=0, \quad \frac{\mathrm{d} v}{\mathrm{~d} t}=0, \\
& g h \frac{\partial(h+b+R)}{\partial x}=0, \quad g h \frac{\partial(h+b+T)}{\partial y}=0,
\end{aligned}
$$

where $R$ and $T$ are primitives to $S_{f x}$ and $S_{f y}$, respectively. Note that the stationary state, the so-called lake at rest, i.e. $u=0=v$, and $h+b=$ const., is a special equilibrium state, that is included here.

Assume that (3.3) holds, then the second equation of (3.1) yields

$$
\begin{aligned}
\frac{g}{2 \hbar^{2}} \int_{y_{i-1 / 2}}^{y_{i+1 / 2}}\left(\left(h_{i+1 / 2}^{n+1 / 2}\right)^{2}-\left(h_{i-1 / 2}^{n+1 / 2}\right)^{2}\right) \mathrm{d} S_{y} & \\
& =\frac{g}{2 \hbar^{2}} \int_{y_{i-1 / 2}}^{y_{i+1 / 2}}\left(h_{i+1 / 2}^{n+1 / 2}+h_{i-1 / 2}^{n+1 / 2}\right)\left(h_{i+1 / 2}^{n+1 / 2}-h_{i-1 / 2}^{n+1 / 2}\right) \mathrm{d} S_{y} .
\end{aligned}
$$

This and the equilibrium condition $g h \partial_{x}(h+b+R)=0$ imply the the well-balanced 
approximation of the source term

$$
\begin{gathered}
\frac{1}{\hbar^{2}} \int_{\Omega_{i j}} B_{2}\left(\boldsymbol{U}^{n+1 / 2}\right)=\frac{1}{\hbar^{2}} \int_{x_{i-1 / 2}}^{x_{i+1 / 2}} \int_{y_{i-1 / 2}}^{y_{i+1 / 2}}-g h^{n+1 / 2}\left(\partial_{x} b^{n+1 / 2}+\partial_{x} R^{n+1 / 2}\right) \\
\approx \frac{-g}{\hbar} \int_{y_{i-1 / 2}}^{y_{i+1 / 2}} \frac{h_{i+1 / 2}^{n+1 / 2}+h_{i-1 / 2}^{n+1 / 2}}{2} \frac{\left(b_{i+1 / 2}+R_{i+1 / 2}^{n+1 / 2}\right)-\left(b_{i-1 / 2}+R_{i-1 / 2}^{n+1 / 2}\right)}{\hbar} \mathrm{d} S_{y} .
\end{gathered}
$$

Integrals along vertical cell interfaces are approximated by the Simpson rule similarly to the cell interface integration used in (3.4). An analogous approximation of the source term is used also in the third equation for the $y$ - direction.

\subsection{Well-balanced approximate evolution operator}

In order to evaluate fluxes on cell interfaces we need to derive approximate evolution operator which give suitable time approximation of the exact integral equations that are implicit in time. The exact integral equations describe time evolution of the solution to the linearized system and can be obtained by exploring the hyperbolic structure of the shallow water system (1.1) and applying the theory of bicharacteristics, cf. [2], [8, 9, 10]. In [12] the well-balanced approximate evolution operators for the shallow water equations with bottom topography have been derived. In [13] we have shown that these operators preserve stationary equilibrium states, i.e. $u=0=v, z=h+b=$ const. Applying the rectangle quadrature for time integrals of the friction terms yields analogously the following approximate evolution operators for the piecewise constant and piecewise bilinear data, cf. [12].

The well-balanced approximate evolution operator $E_{\Delta}^{\text {const }}$ for piecewise constant data reads

$$
\begin{aligned}
h(P)= & \frac{1}{2 \pi} \int_{0}^{2 \pi}(h(Q)+b(Q))-\frac{\tilde{c}}{g} u(Q) \operatorname{sgn}(\cos \theta)-\frac{\tilde{c}}{g} v(Q) \operatorname{sgn}(\sin \theta) \mathrm{d} \theta \\
& -b(P)+\frac{\tilde{c} \Delta t}{2 \pi} \int_{0}^{2 \pi}\left(S_{f x}(Q) \cos \theta+S_{f y}(Q) \sin \theta\right) \mathrm{d} \theta+O\left(\Delta t^{2}\right) \\
u(P)= & \frac{1}{2 \pi} \int_{0}^{2 \pi}-\frac{g}{\tilde{c}}(h(Q)+b(Q)) \operatorname{sgn}(\cos \theta)+u(Q)\left(\cos ^{2} \theta+\frac{1}{2}\right) \\
& +v(Q) \sin \theta \cos \theta d \theta-g \Delta t S_{f x}\left(Q_{0}\right)+O\left(\Delta t^{2}\right), \\
v(P)= & \frac{1}{2 \pi} \int_{0}^{2 \pi}-\frac{g}{\tilde{c}}(h(Q)+b(Q)) \operatorname{sgn}(\sin \theta)+u(Q)(\sin \theta \cos \theta) \\
& +v(Q)\left(\sin ^{2} \theta+\frac{1}{2}\right) d \theta-g \Delta t S_{f y}\left(Q_{0}\right)+O\left(\Delta t^{2}\right) .
\end{aligned}
$$

If the continuous piecewise bilinear data are used the well-balanced approximate evolution 
operator, which is denoted by $E_{\Delta}^{\text {bilin }}$, reads

$$
\begin{aligned}
h(P)= & -b(P)+\left(h\left(Q_{0}\right)-b\left(Q_{0}\right)\right)+\frac{1}{4} \int_{0}^{2 \pi}\left(\left(h(Q)-h\left(Q_{0}\right)\right)+\left(b(Q)-b\left(Q_{0}\right)\right)\right) \mathrm{d} \theta \\
& -\frac{1}{\pi} \int_{0}^{2 \pi}\left(\frac{\tilde{c}}{g} u(Q) \cos \theta+\frac{\tilde{c}}{g} v(Q) \sin \theta\right) \mathrm{d} \theta \\
& +\frac{\tilde{c} \Delta t}{2 \pi} \int_{0}^{2 \pi}\left(S_{f x}(Q) \cos \theta+S_{f y}(Q) \sin \theta\right) \mathrm{d} \theta+O\left(\Delta t^{2}\right), \\
u(P)= & u\left(Q_{0}\right)-\frac{1}{\pi} \int_{0}^{2 \pi} \frac{g}{\tilde{c}}(h(Q)+b(Q)) \cos \theta \mathrm{d} \theta \\
& +\frac{1}{4} \int_{0}^{2 \pi}\left(3 u(Q) \cos \theta+3 v(Q) \sin \theta \cos \theta-u(Q)-\frac{1}{2} u\left(Q_{0}\right)\right) \mathrm{d} \theta \\
& -g \Delta t S_{f x}\left(Q_{0}\right)+O\left(\Delta t^{2}\right), \\
& v\left(Q_{0}\right)-\frac{1}{\pi} \int_{0}^{2 \pi} \frac{g}{\tilde{c}}(h(Q)+b(Q)) \sin \theta \mathrm{d} \theta \\
& +\frac{1}{4} \int_{0}^{2 \pi}\left(3 u(Q) \sin \theta \cos \theta+3 v(Q) \sin { }^{2} \theta-v(Q)-\frac{1}{2} v\left(Q_{0}\right)\right) \mathrm{d} \theta \\
& -g\left(Q_{0}\right)+O\left(\Delta t^{2}\right) .
\end{aligned}
$$

The approximate evolution operators (3.5) and (3.6) are used in (3.2) in order to evolve fluxes along cell interfaces. Thus, the first order method is obtained using the approximate evolution operator $E_{\Delta}^{\text {const }}$

$$
\overline{\boldsymbol{f}}_{k}^{n+1 / 2}=\frac{1}{\hbar} \int_{\mathcal{E}} \boldsymbol{f}_{k}\left(E_{\Delta t / 2}^{\text {const }} \boldsymbol{U}^{n}\right) \mathrm{d} S, \quad k=1,2,
$$

whereas in the second order FVEG scheme a suitable combination of the approximate evolution operator $E_{\Delta}^{\text {bilin }}$ and $E_{\Delta}^{\text {const }}$ is used. We apply $E_{\Delta}^{\text {bilin }}$ to evolve slopes and $E_{\Delta}^{\text {const }}$ to evolve the corresponding constant part in order to preserve conservativity

$$
\overline{\boldsymbol{f}}_{k}^{n+1 / 2}=\frac{1}{\hbar} \int_{\mathcal{E}} \boldsymbol{f}_{k}\left(E_{\Delta t / 2}^{\text {bilin }} R_{h} \boldsymbol{U}^{n}+E_{\Delta t / 2}^{\text {const }}\left(1-\mu_{x}^{2} \mu_{y}^{2}\right) \boldsymbol{U}^{n}\right) \mathrm{d} S .
$$

Here $R_{h} \boldsymbol{U}$ denotes a continuous bilinear recovery and $\mu_{x}^{2} U_{i j}=1 / 4\left(U_{i+1, j}+2 U_{i j}+U_{i-1, j}\right)$; an analogous notation is used for the $y$-direction.

\section{$4 \quad$ Numerical experiments}

In this section we compare the behavior of both FEM and FVEG schemes through several test problems. 


\section{Example 1: a channel flow with friction}

In this example we simulate a steady uniform flow in a regular rectangular channel of $\ell=1 \mathrm{~km}$ length and $w=6 \mathrm{~m}$ width. The bottom profile is given by

$$
b(x, y)=-0.001 x+1 \quad 0<x<1000, y \in[0,6] .
$$

For the FVEG method the initial data are chosen as a stationary state

$$
h(x, y, 0)+b(x, y)=2, u(x, y, 0)=0=v(x, y, 0) .
$$

At the inflow, i.e. $x=0 m$, the volume rate flow is taken to be $Q \equiv w h u=3 m^{3} s^{-1}$. The inflow velocity in the $y$ - direction is $0 \mathrm{~ms}^{-1}$. At the outflow, i.e. $x=1000 \mathrm{~m}$, we have imposed for the FVEG method absorbing boundary conditions by extrapolating the data in the outer normal direction. We have tested several friction parameters of the bottom, $k_{s}=0.1,0.2$ and 0.3 . In order to evaluate friction slopes the hydraulic radius $r_{h y}$ is to be computed. For a regular rectangular channel it is computed by the formula $r_{h y}=w h /(2 h+b)$.

Solutions computed by the FVEG method is evolved in time until the steady equilibrium state is achieved, i.e. until $\left\|h^{n+1}-h^{n}\right\| \leq 10^{-8}$. Since the FVEG method is explicit in time, the CFL stability condition needs to be satisfied. We set CFL number to 0.8 in all our experiments. The FVEG method (3.1) solves two-dimensional shallow water equations (1.1), the solution in the $y$-direction is constant.

The FEM computes directly solution of the stationary equations, i.e. $\frac{\partial \boldsymbol{w}}{\partial t}=0$ in (1.2), i.e. (2.2) and (2.3). The FEM method (2.5), (2.6) approximates the one-dimensional SaintVenant equations (1.2), i.e. (2.2), (2.3). The comparisons of the shallow water depths are given for different values of $k_{s}$ in the Table 1 . The results indicate clearly very good agreement of both methods, the second order FVEG scheme as well as FEM.

\begin{tabular}{|l|l|l|}
\hline \multirow{2}{*}{$k_{s}$} & \multicolumn{2}{|c|}{$h[m]$} \\
\cline { 2 - 3 } & FEM & FVEG \\
\hline 0.1 & 0.6395567 & 0.640179 \\
\hline 0.2 & 0.7113167 & 0.712090 \\
\hline 0.3 & 0.7626931 & 0.763592 \\
\hline
\end{tabular}

Table 1: Comparison of water depths for a steady flow with different friction parameters for the bottom.

\section{Example 2: a channel flow with friction and varied topography}

In this example we simulate again a steady flow in a channel having a varying bottom topography. We take a non smooth bottom having discontinuity in the first derivative, the profile is given as

$$
b(x, y)= \begin{cases}-0.001(x-500)+0.5 & \text { if } 0<x<500, y \in[0,6] \\ 0.5 & \text { if } 500<x<1000, y \in[0,6] .\end{cases}
$$

The length of the channel is $\ell=1 \mathrm{~km}$ and width is $w=6 \mathrm{~m}$. The grain roughness size parameter of the bottom is set to $k_{s}=0.1$. We take again for the FVEG a stationary state as the initial data, i.e. $h(x, y, 0)+b(x, y)=2, u(x, y, 0)=0=v(x, y, 0)$. Other parameters, inflow and outflow boundary conditions, are the same as in the previous 
experiment. The solution is evolved in time until a steady state is obtained. Our steady state solutions obtained by different methods, i.e. the FVEG method as well as the FEM are in a very good agreement, see Figures 1. No singular corner effects on smoothness of the solution can be noticed.
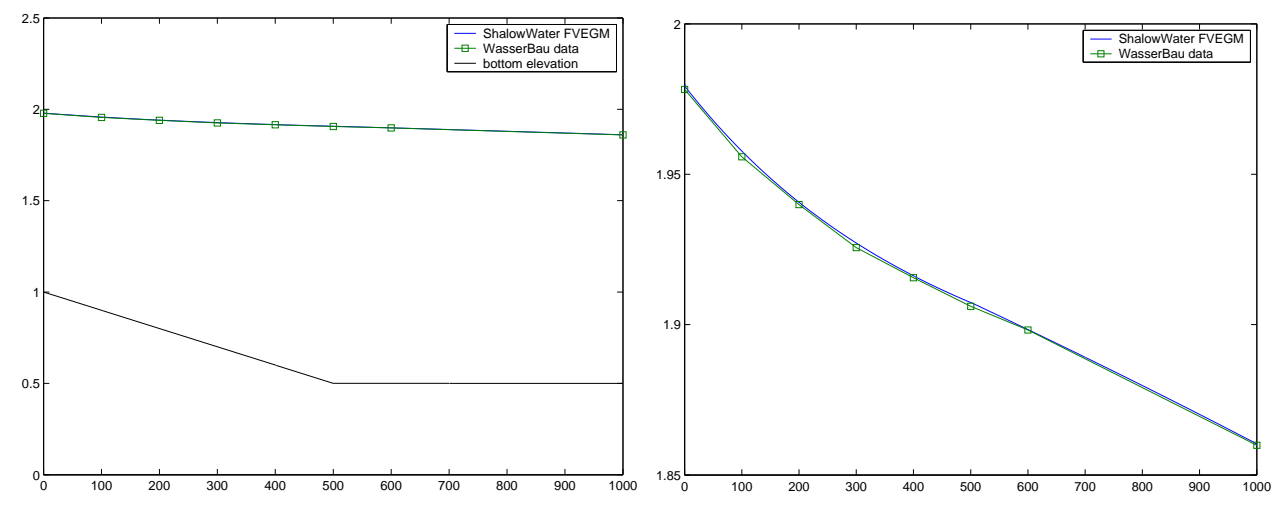

Figure 1: Comparison of the two-dimensional solution obtained by the FVEG scheme (solid line) and the one-dimensional steady solution obtained by the FEM scheme (boxes).

\section{Example 3: propagating waves with a bottom topography}

In this example small perturbances of a stationary flow are simulated. It is well-known that this is a hard test for methods which do not take care on a well-balanced approximation of the bottom topography and friction terms. In this case strong oscillations can appear in solutions as soon as small perturbations propagate over topographical changes. We consider here a problem analogous to that proposed by LeVeque, cf.[7].

The bottom topography consists of one hump

$$
b(x)= \begin{cases}0.25(\cos (10 \pi(x-0.5))+1) & \text { if }|x-0.5|<0.1 \\ 0 & \text { otherwise }\end{cases}
$$

and the initial data are $u(x, 0)=0$,

$$
h(x, 0)= \begin{cases}1-b(x)+\varepsilon & \text { if } 0.1<x<0.2 \\ 1-b(x) & \text { otherwise. }\end{cases}
$$

The parameter $\varepsilon$ is chosen to be 0.2 and 0.02 . The computational domain is $[0,100]$. The reflection boundary conditions, i.e. fixed wall conditions, have been imposed on $x=0 \mathrm{~m}$ and $x=100 \mathrm{~m}$. In the FEM the Dirichlet boundary conditions, i.e. $Q=0$, are imposed, which is an alternative to model fixed walls. The value of $k_{s}$ is set to $0.1 \mathrm{~m}$.

Firstly the perturbation parameter is taken to be $\varepsilon=0.2$. In Figures 2,3,4 we can see propagation of small perturbances of the water depth $h$ for different times until $t=19$ seconds. Solution is computed on a mesh with 500 cells with the second order FVEG method as well as FEM.

The FVEG method uses a time step $\Delta t$ computed directly according to the CFL condition, $C F L=0.8$. In the FEM time step $\Delta t$ was set to $\Delta t=0.015$ for $\varepsilon=0.2$ and $\Delta t=0.02$ for $\varepsilon=0.02$. For $\varepsilon=0.02$ the time step is of the same order as the time step used by the FVEG method. In the case $\varepsilon=0.2$ the time step given automatically by the CFL condition is $\Delta t \approx 0.18$. In this case we have decided to suppress the time step for the FEM in order to reduce its numerical diffusion; as mentioned above $\Delta t=0.02$ was used. In our numerical experiments we have seen that it was enough to take approximately 10 inner iterations in the Newton-Raphson method. 
In Figure 5,6,7 the perturbation parameter is $\varepsilon=0.02$, which is of the order of the discretization error being $O\left(10^{-2}\right)$. We can notice correct resolution of small perturbances of the steady state by both methods even if the perturbances are of the order of the truncation error. Small initial oscillations can be noticed in the FEM, which is also slightly more dissipative than FVEG scheme. Since the flow is relatively slow, the Froude number is less that 1, no upwinding technique was necessary in the FEM. It would be however important to stabilize the finite element approximation by some type of upwinding technique or streamline diffusion technique, if flows with higher Froude numbers will be modelled. The FVEG method is constructing in such a way that it exactly balances influence of fluxes and the source terms. Although we have used just standard finite element approximation in the case of our FEM method, presented in the Chapter 2, no unbalanced oscillations can be noticed as perturbed waves propagate over the bottom topography even for small perturbations. The reason is the formulation (2.3) where we have modeled the water level $z$, instead of separating height $h$ and topography $b$. Note also that the FEM discretizes also the friction term in the same way as the water level $z$. Thus the equilibrium condition (3.3) is preserved here as well.

For a two-dimensional analogy of this experiment similar results have been obtained by the FVEG method, cf. [13], which is a trully multidimensional scheme. The approach presented here, which is based on the FEM, is designed only for one-dimensional shallow flows, i.e. Saint Venant equations (2.2), (2.3). The generalization to fully two-dimensional case is possible as well. In practice the one-dimensional FEM computation of nearly onedimensional river flows are often satisfactory and moreover more effective and less time consuming.

\section{Concluding remarks}

In the present paper we have compared two different discretization techniques for approximation of the system of shallow water equations with bottom topography terms and friction terms. The first method, the FEM, is based on a classical finite element approach using conforming linear finite elements for approximation of water depth $h$ and for the volume rate $Q$. Discretization in time is done by the backward Euler scheme yielding the implicit finite difference scheme in time. The resulting nonlinear system of algebraic differential equations is solved iteratively by the Newton Raphson method. It should be pointed out that the presented scheme have already been used successfully for real river flow calculations in practice.

The second method, the finite volume evolution Galerkin method (FVEG), belongs to the class of multidimensional finite volume methods and it is a genuinely multidimensional variant of classical finite volume schemes. Thus, the FVEG method is a time explicit scheme and it resolves correctly also strong (multidimensional) shocks due to its upwinding character, cf. [8], [9], [10]. In order to obtain a higher order resolution a recovery step is included in the computation of cell-interface fluxes. It should be pointed out that in contrast to the finite element scheme, the FVEG method uses discontinuous (bi-)linear discrete functions. Thus, the FEM as well as the FVEG scheme are second order accurate in space.

In order to resolve correctly steady equilibrium states a special, the so-called well-balanced, approximation of terms modelling the bottom topography as well as the friction effects was necessary. The FEM approximates these terms directly in the same way as other momentum terms and no special approximation was implemented. Both principally dif- 
ferent discretization schemes have been extensively tested on various one-dimensional test problems, the representative choice of them is presented here. Surprisingly enough, we have found in all our experiments an excellent agreement of both methods. Only relatively marginal differences can be found even on hard well-balanced problems, cf. Example 3. As far as the CPU time concerns we can noticed that the time explicit FVEG scheme was generally faster than the implicit FEM. However, the CPU-efficiency needs to be consider relatively since it depends on the optimality and robustness of a code. We think that our comparative study can initiate an interest of engineers, who deal with the river flow simulations, to use new modern methods coming from other fields, such as the FEM and the FVEG method presented here.

It is fair to mention that we have not yet deal with other interesting problems like the resonance phenomenon and roll waves. In the case of resonance phenomenon two eigenvalues of the propagation matrix (Jacobian matrix) collapse. In the shallow water system with topography the resonance phenomenon appears when speed of gravity waves vanishes. Assume for example a decresing topography, then the fluvial (subcritical) flow can change to the torrential (supercritical) flow through a stationary shock, the so-called hydraulic jump, the Bernoulli's law can be violated and the uniqueness of the weak entropy solutions is lost.

Further, it is known that roll waves can occur in a uniform open-channel flow down an incline, when the Froude number is larger than two. It has been shown by [18] that the initial value problem for the Saint-Venant system including topography and friction is then linearly unstable. For steep channels the uniform flow can break to a series of waves or bores that are separated by smooth flow in a staircase pattern. These are the roll waves, i.e. discontinuous periodic travelling waves. The reliable and robust numerical schemes should produce correct approximations of these complex situations, too. This is a topic for further study.

\section{Appendix}

For the Newton-Raphson scheme equations (2.5) and (2.6) has to be differentiated. The corresponding derivatives, which are the entries of the Jacobian matrix in (2.9) are for each node $i=1, \ldots, n:$ :

$$
\begin{gathered}
\frac{\partial G}{\partial Q_{i}}=\int_{0}^{L} N^{T}\left(\frac{\mathrm{d} N_{i}}{\mathrm{~d} x}\right) \mathrm{d} x \\
\frac{\partial G}{\partial h_{i}}=\int_{0}^{L} N^{T} N_{i}\left(\frac{\partial^{2} A}{\partial h^{2}} \frac{\partial h}{\partial t}+\frac{\partial A}{\partial h} \frac{\gamma}{\Delta t}\right) \mathrm{d} x \\
\frac{\partial S_{f}}{\partial h_{i}}=-2 N_{i} \frac{S_{E} Q^{2}}{Q_{S c h}^{3}}\left(\frac{\partial Q_{S c h}}{\partial h}\right) \\
\frac{\partial F}{\partial Q_{i}}=\int_{0}^{L} N^{T} N_{i}\left(\frac{\gamma}{\Delta t}+\frac{2 Q}{A} \frac{\partial \beta}{\partial x}+\frac{2 \beta}{A}\left(\frac{\partial Q}{\partial x}+\frac{Q}{N_{i}} \frac{\partial N_{i}}{\partial x}\right)-2 \frac{\beta Q}{A^{2}} \frac{\partial A}{\partial x}+2 g \frac{A}{Q} S_{f}\right) \mathrm{d} x .
\end{gathered}
$$


Here $Q_{S c h}$ describes the stage flow relationship for each cross section for steady uniform flow with the bottom slope $S_{E}$. In the described model we have approximated this relationship by polynomials in a preprocessing process. If there is no discharge $Q=0$ than the last term of (6.4) vanishes, i.e.

$$
2 g \frac{A}{Q} S_{f}=0
$$

for more details see [17].

$$
\begin{aligned}
\frac{\partial F}{\partial h_{i}}= & \int_{0}^{L}\left\{N ^ { T } \left[N_{i} \frac{Q^{2}}{A^{2}}\left(A \frac{\partial^{2} \beta}{\partial h \partial x}-\frac{\partial \beta}{\partial x} \frac{\partial A}{\partial x}\right)+N_{i} \frac{2 Q}{A^{2}} \frac{\partial Q}{\partial x}\left(A \frac{\partial \beta}{\partial h}-\beta \frac{\partial A}{\partial h}\right)\right.\right. \\
& -N_{i} \frac{Q^{2}}{A^{2}}\left(\frac{\partial \beta}{\partial h} \frac{\partial A}{\partial x}+\beta \frac{\partial^{2} A}{\partial h \partial x}-2 \frac{\beta}{A} \frac{\partial A}{\partial h} \frac{\partial A}{\partial x}\right) \\
& \left.\left.+g\left(N_{i} \frac{\partial A}{\partial h} \frac{\partial h}{\partial x}+A \frac{\partial N_{i}}{\partial x}\right)+g N_{i}\left(S_{f} \frac{\partial A}{\partial h}+A \frac{\partial S_{f}}{\partial h}\right)-g N_{i} S_{0}\left(\frac{\partial A}{\partial h}\right)\right]\right\} \mathrm{d} x
\end{aligned}
$$

In the examples presented in the paper the momentum coefficient $\beta$ was set to unity and no lateral inflow $q_{e}$ was considered.

We would like to point out that for arbitrary cross sections $A$ the following relationship is often used

$$
\frac{\partial A}{\partial h_{i}}=N_{i}(x) \frac{\partial A}{\partial h}
$$

see [17] for more detailed discussion.

\section{Acknowledgements}

The research of the first author was partially supported by the Graduate College 'Conservation principles in the modelling and simulation of marine, atmospherical and technical systems' of Deutsche Forschungsgemeinschaft. She would like to thank Professor Erik Pasche, TU Hamburg-Harburg, for initiating this coopeartion.

\section{References}

[1] N. Botta, R. Klein, S. Langenberg, S. Lützenkirchen, Well balanced finite volume methods for nearly hydrostatic flows, J. Comp. Phys., 196(2004), pp. 539-565.

[2] R. Courant, D. Hilbert, Methoden der mathematischen Physik II, second edition, Springer, Berlin, 1968

[3] M. Fey, Multidimensional upwinding, Part II. Decomposition of the Euler equations into advection equations, J. Comp. Phys., 143(1998), pp. 181-199.

[4] J.M. Greenberg, A.-Y. LeRoux, A well-balanced scheme for numerical processing of source terms in hyperbolic equations, SIAM J. Numer. Anal., 33(1996), pp. 1-16. 
[5] A. Kurganov, D. Levy, Central-upwind schemes for the Saint-Venant system, $\mathrm{M}^{2} \mathrm{AN}$, Math. Model. Numer. Anal., 36(3)(2002), pp. 397-425.

[6] R.J. LeVEQUE, Wave propagation algorithms for multidimensional hyperbolic systems, J. Comp. Phys., 1997(131), pp. 327-353.

[7] R.J. LeVEque, Balancing source terms and flux gradients in high-resolution Godunov methods: The quasi-steady wave propagation algorithm, J. Comp. Phys., 146(1998), pp. 346-365.

[8] M. LukÁČová-MedviĎová, J. Saibertová, Genuinely multidimensional evolution Galerkin schemes for the shallow water equations, Enumath Conference, in Numerical Mathematics and Advanced Applications, F. Brezzi et al., eds., World Scientific Publishing Company, Singapore, 2002, pp. 105-114.

[9] M. Lukáčová-Medviďová, J. Saibertová, G. Warnecke, Finite volume evolution Galerkin methods for nonlinear hyperbolic systems, J. Comp. Phys., 183(2002), pp. 533-562.

[10] M. LukÁčová-MedviĎová, K.W. Morton, G. Warnecke, Finite volume evolution Galerkin methods for hyperbolic problems, SIAM J. Sci. Comput. 26(1)(2004), pp. 1.-30.

[11] I. KING, Finite element models for unsteady flow routing through through irregular channels, Proc. first int. conference on FE in Waterresources, Pentech Press, London,(1976), pp. 166-184.

[12] M. LuKÁČOVÁ-MedviĎOvÁ, Z. VlK, Well-balanced finite volume evolution Galerkin methods for the shallow water equations with source terms, Int. J. Numer. Meth. Fluids, 2004, accepted.

[13] M. LuKÁČOvÁ-MedviĎová, S. Noelle, Well-balanced finite volume evolution Galerkin methods for the shallow water equations with source terms modelling bottom topography and Coriolis forces, in preparation.

[14] S. NOELLE, The MOT-ICE: a new high-resolution wave-propagation algorithm for multidimensional systems of conservative laws based on Fey's method of transport, J. Comp. Phys. 164(2000), pp. 283-334.

[15] J. SHI, A steady-state capturing method for hyperbolic systems with geometrical source terms, M²AN, Math. Model. Numer. Anal., 35(4)(2001), pp. 631-646.

[16] U. TESCHKE, A new procedure of solving the one-dimensional Saint-Venant equations for natural rivers, in Wasserbau fünf Jahre, E.Pasche, ed., TU Hamburg-Harburg, 2003, pp. 35-39.

[17] U. TESCHKE, Zur Berechnung eindimensionaler instationärer Strömungen von natürlichen Fließgewässern mit der Methode der Finiten Elemente, PhD Dissertation TU Hamburg-Harburg, 2003, Fortschritt-Berichte VDI, 7/ 458, 2004.

[18] J. Whiтham, Linear and Nonlinear Waves, Wiley, 1974.

[19] B.C. YEN, Open channel flow resistance, J. Hydraulic Eng. 128(2002), pp. 20-39 

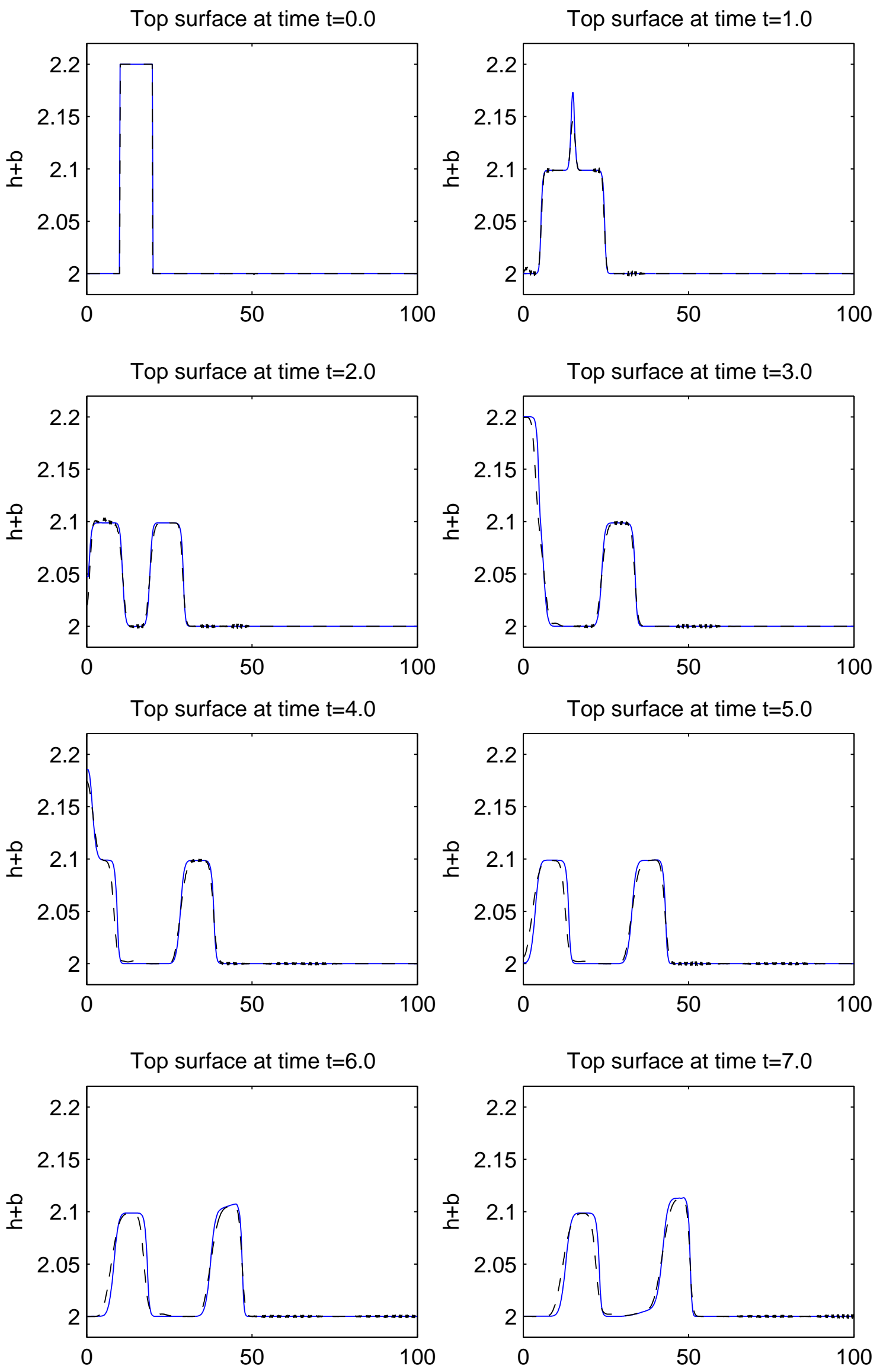

Figure 2: Propagation of small pertutbances, $\varepsilon=0.2, T=0,1, \ldots 7$ seconds; computed by the FVEG method (solid line) and by the FEM (dotted line). 

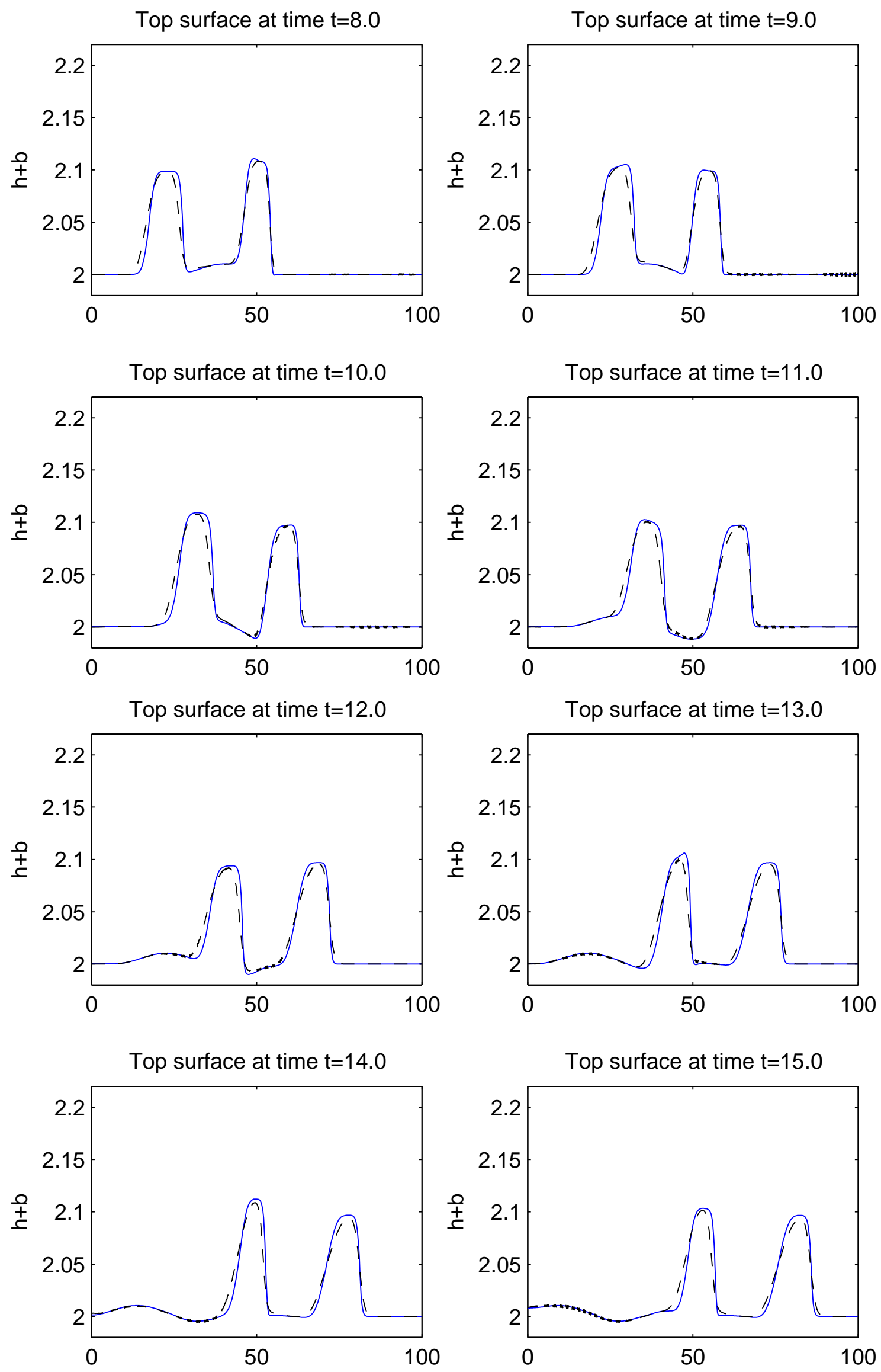

Figure 3: Propagation of small pertutbances, $\varepsilon=0.2, T=8,9, \ldots 15$ seconds; computed by the FVEG method (solid line) and by the FEM (dotted line). 

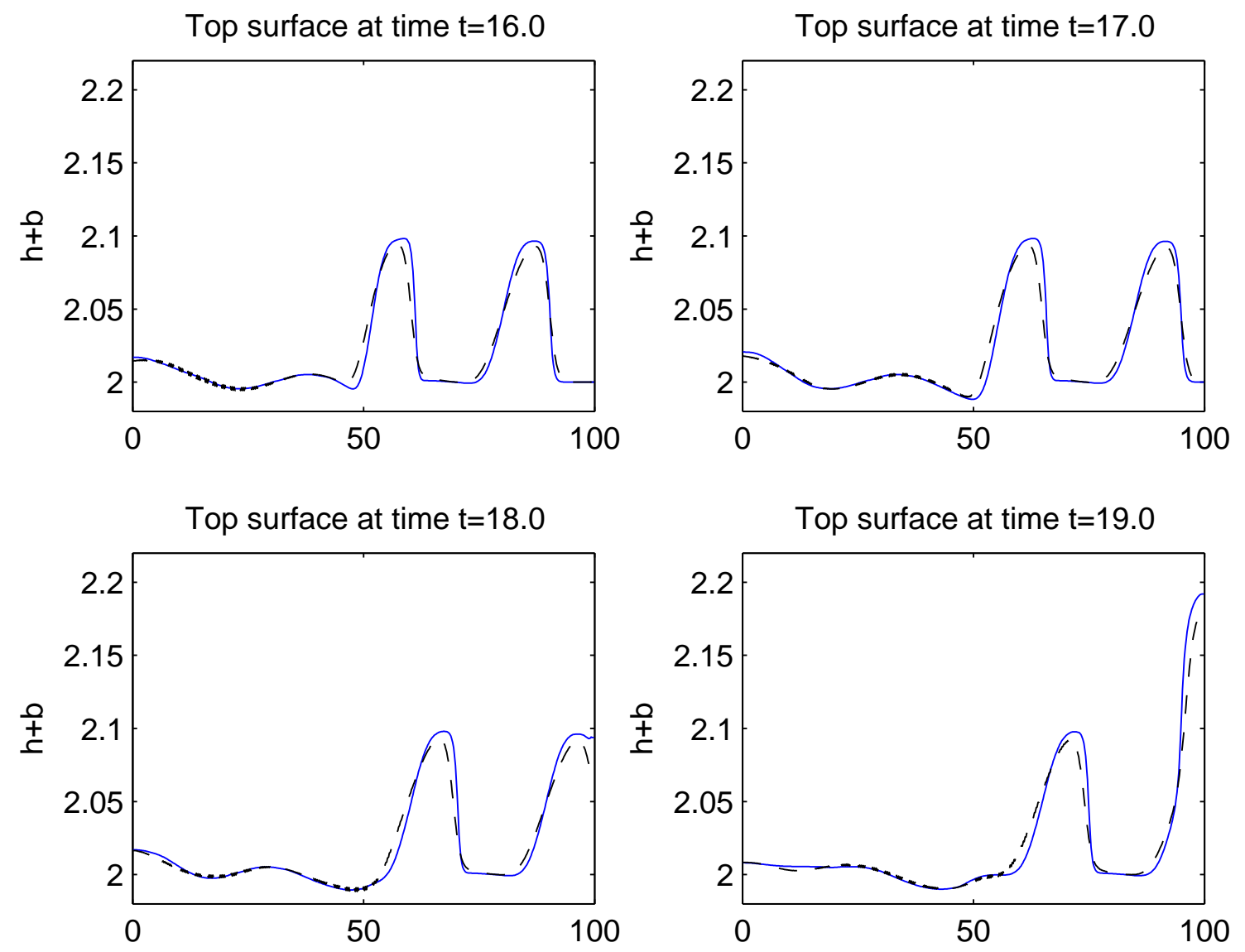

Figure 4: Propagation of small pertutbances, $\varepsilon=0.2, T=16,17,18,19$ seconds; computed by the FVEG method (solid line) and by the FEM (dotted line). 

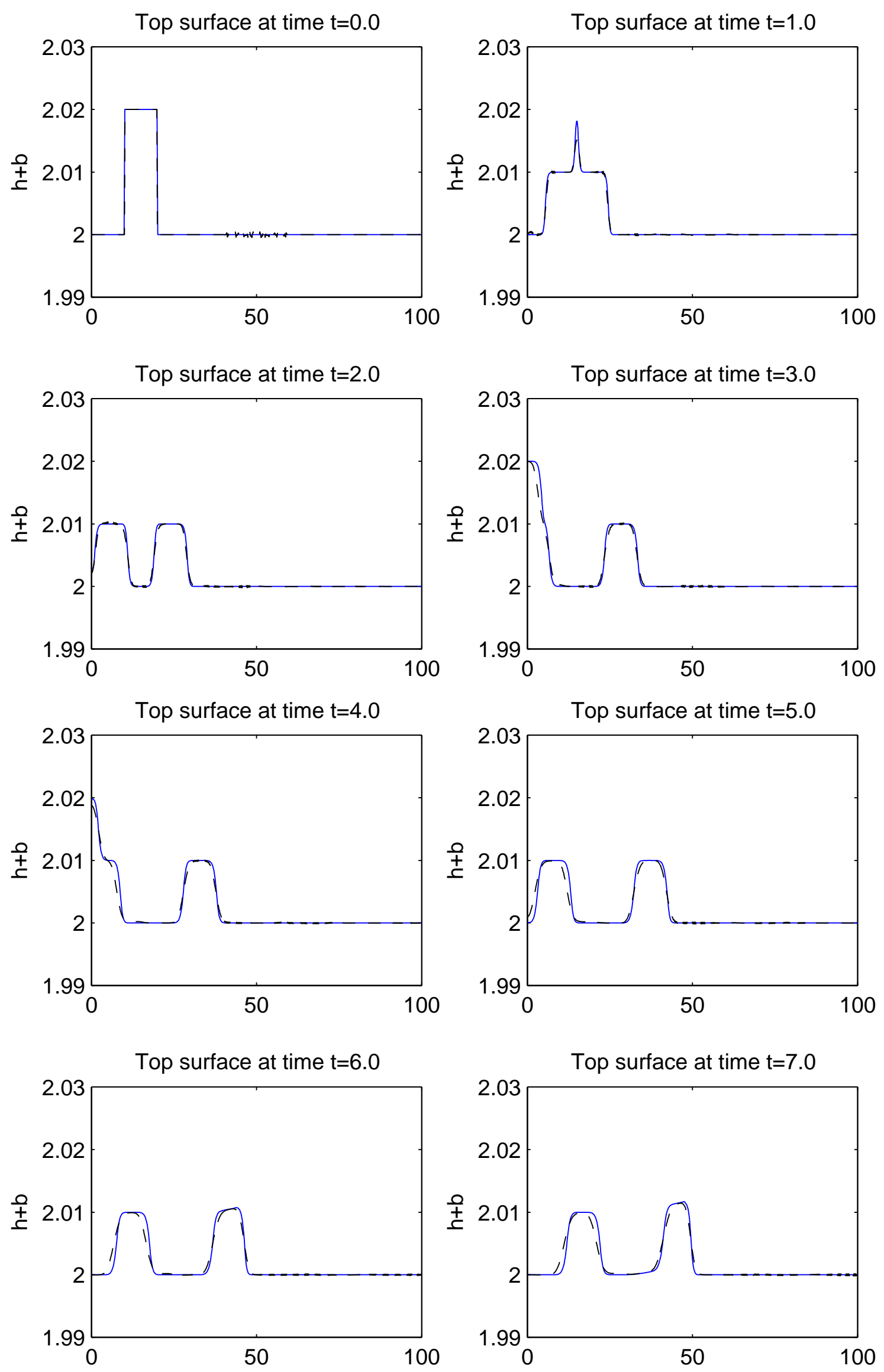

Figure 5: Propagation of small pertutbances, $\varepsilon=0.02, T=0,1, \ldots 7$ seconds; computed by the FVEG method (solid line) and by the FEM (dotted line). 

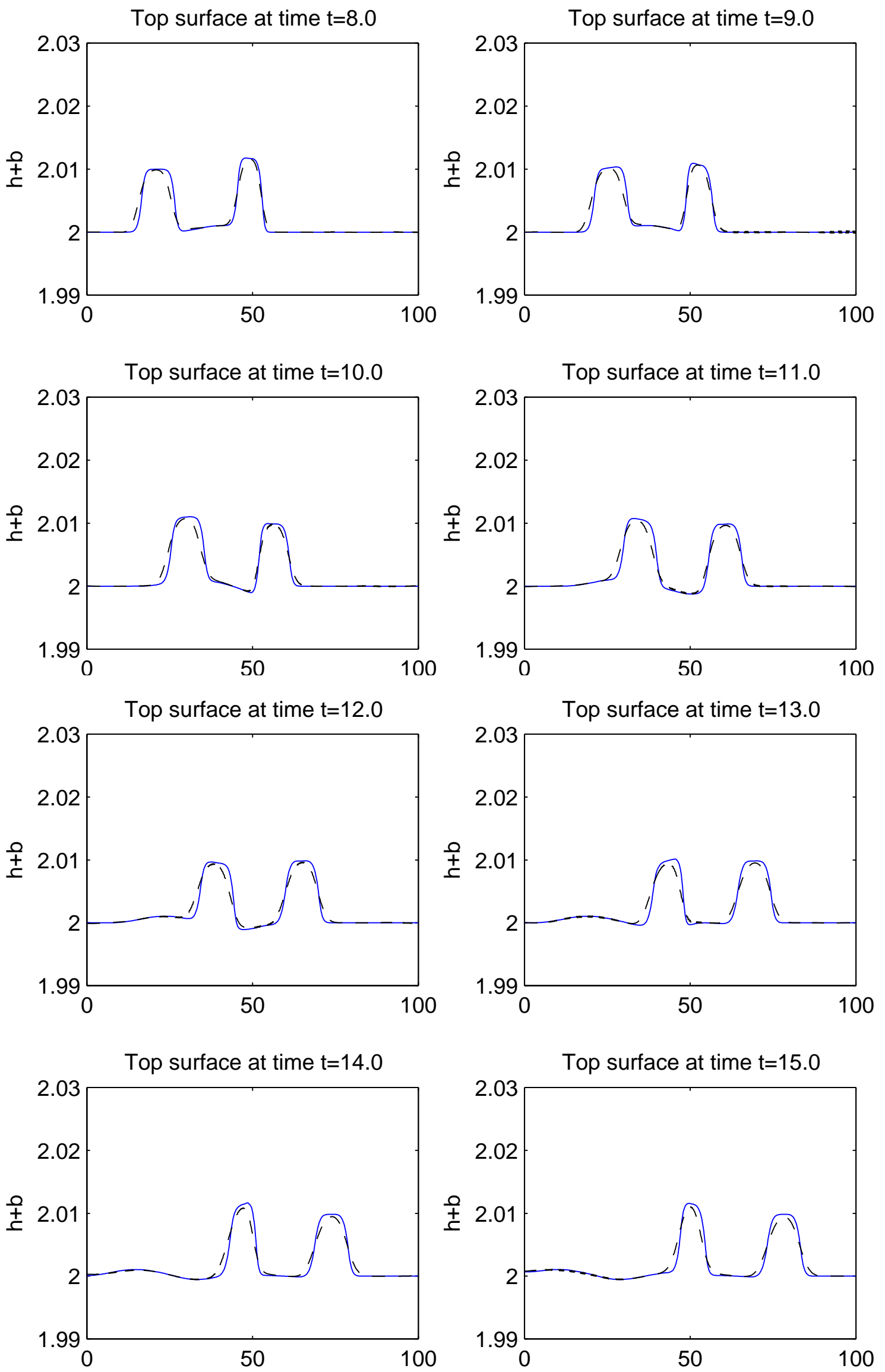

Figure 6: Propagation of small pertutbances, $\varepsilon=0.02, T=8,9, \ldots 15$ seconds; computed by the FVEG method (solid line) and by the FEM (dotted line). 

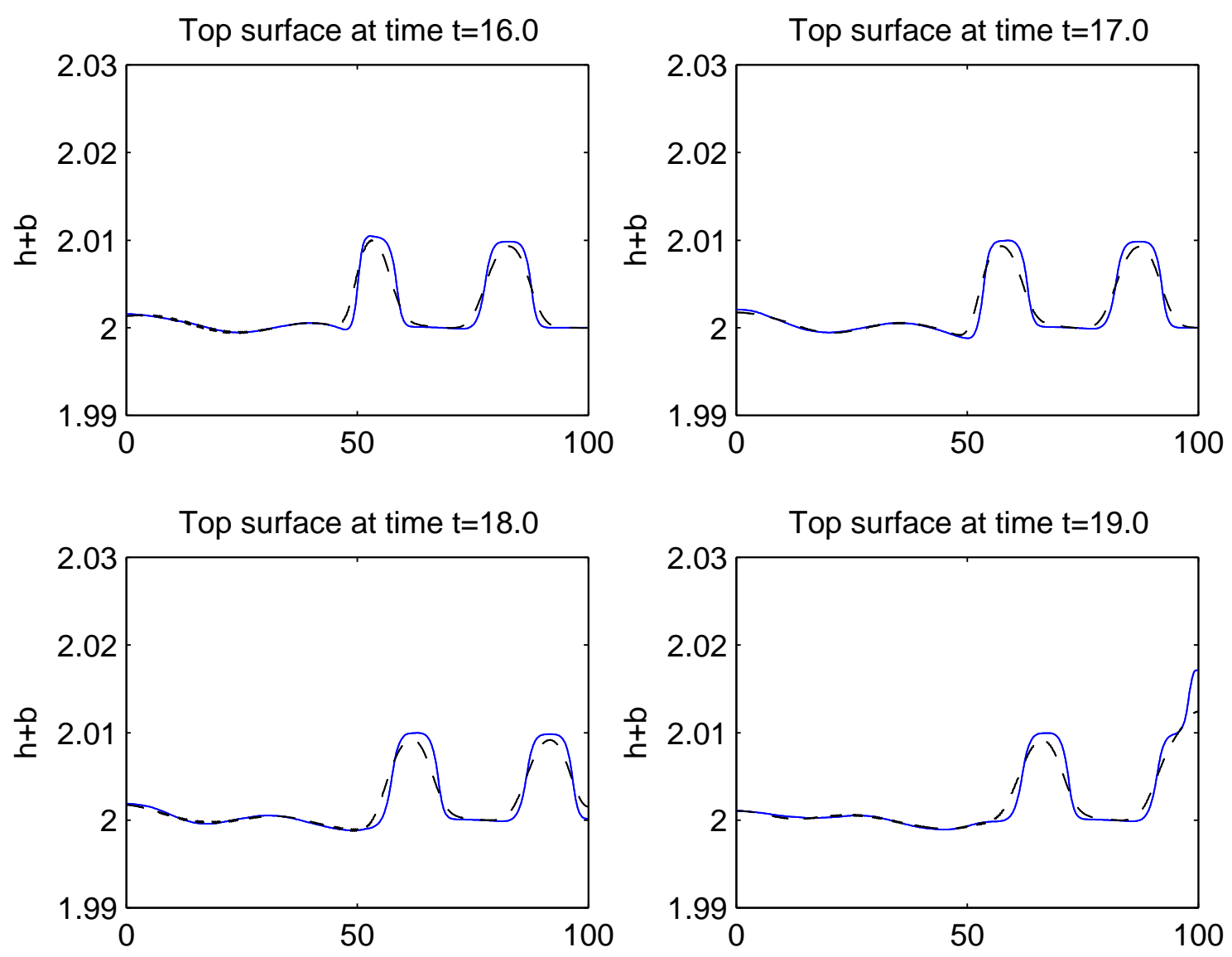

Figure 7: Propagation of small pertutbances, $\varepsilon=0.02, T=16,17,18,19$ seconds; computed by the FVEG method (solid line) and by the FEM (dotted line). 